\section{CONFIGURACIÓN DEL RIESGO SOCIO NATURAL DE LOS ALGUEROS DE LA LANCHA, EN BUCALEMU, PAREDONES*}

Daniela Martínez Olivares**

Carlos Humberto Muñoz Parra***

\section{Resumen}

Esta investigación analiza las experiencias y conocimientos en torno al riesgo socio-natural que surgen de las dinámicas del hábitat residencial de los extractores de algas de Bucalemu, Región de O’Higgins. El habitar en riesgo, por la probabilidad de eventos tales como un tsunami o marejadas, configura gestiones espontáneas al riesgo socionatural y capacidades adaptativas. Estos eventos son el resultado de experiencias durante más de 80 años en el territorio. La comprensión de estas gestiones y capacidades se orientan a contribuir desde una mirada integral a las medidas de mitigación del riesgo socio natural.

\section{EMERGENCE OF SOCIO- NATURAL RISK FOR SEAWEED COLLECTORS IN BUCALEMU, PAREDONES*}

\author{
Daniela Martínez Olivares**, \\ Carlos Humberto Muñoz Parra***
}

\section{Abstract}

This research analyzes the experiences and knowledge of the socio-natural risks resulting from the dynamics of residential habitat for seaweed collectors in Bucalemu, Region of O'Higgins. Given the probability of the occurrence of tsunamis or sea surges, the endangered habitat generates spontaneous measures in relation to socio-natural risk and adaptive capacities, the result of more than 80 years of experience within this territory. The understanding of these management and capacities contribute to the elaboration of mitigation measures to face socionatural risks. 


\section{PALABRAS CLAVES: DESASTRES, RIESGO SOCIO NATURAL, ALGUEROS, SISTEMAS SOCIO- ECOLÓGICOS, ADAPTABILIDAD.}

Recibido: 09-06-2015

Aceptado: 17-05-2016

* El presente artículo expone un resumen de la investigación realizada para obtener el grado de Magister en Hábitat Residencial, del Instituto de la Vivienda, de la Facultad de Arquitectura y Urbanismo de la Universidad de Chile.

* Chile. Arquitecta Pontificia Universidad Católica de Valparaíso. Magister en Habitat Residencial. Arquitecto en el Equipo de Rehabilitación Urbana del Servicio de Vivienda y Urbanismo en la Región Metropolitana. Correo electrónico: arquitecta.dmo@ gmail.com

** Chile. Arquitecto por la Pontificia Universidad Católica de Chile; Doctor en Arquitectura en la Escuela Técnica Superior de Arquitectura de la Universidad Politécnica de Madrid. Profesor Escuela de Arquitectura de la Universidad de Santiago de Chile. Correo electrónico: cmparquitecto@yahoo.es
KEYWORDS: DISASTERS, SOCIO-NATURAL RISK, SEAWEED COLLECTORS, SOCIO-ECOLOGICAL SYSTEMS, ADAPTABILITY.

Received: 09-06-2015

Accepted: 17-05-2016

* This paper summarizes the Master's Degree thesis conducted by the author to in order to obtain an MSc in Residential Habitat, the Housing Institute at the Faculty of Architecture and Urban Planning, University of Chile.

** Chile. Architect, Pontifical Catholic University of Chile. MSc in Residential Habitat. Member of the Urban Rehabilitation Group at the Housing and Development Service, Metropolitan Region. Email: arquitecta.dmo@gmail.com

*** Chile. Architect, Pontifical Catholic University of Chile; PhD in Architecture, Higher Technical School of Architecture at the Technical University of Madrid. Professor, School of Architecture, University of Santiago, Chile. Email: cmparquitecto@yahoo.es 


\section{Introducción}

El presente artículo muestra los resultados de una investigación llevada a cabo para obtener la Maestría en Hábitat Residencial que imparte el Instituto de la Vivienda de la Universidad de Chile. Tiene un carácter exploratorio-descriptivo, en donde se busca conocer, comprender y analizar las dinámicas del hábitat residencial de grupos rurales que dependan económicamente de los recursos naturales y su renovación (Sistemas socio-ecológicos) que están expuestos a riesgos socio-naturales. De esta manera, se intenta responder cómo estos grupos rurales se adaptan a las amenazas y desastres naturales, a los que se ven expuestos. Esto, con el fin de contribuir desde una mirada holística a las medidas de prevención, mitigación y reconstrucción de su hábitat residencial ante la eventualidad de un desastre socio natural. Se aborda desde la experiencia personal trabajando para la reconstrucción post terremoto y tsunami del 2010 en la zona de Cardenal Caro, con familias damnificadas, coordinando la ejecución de subsidios de autoconstrucción de viviendas, gestión realizada en el Servicio de Vivienda y Urbanismo de la Región de O'Higgins, desde finales del 2011 hasta comienzos del 2013.

La muestra del estudio corresponde a un grupo de familias de extractores de algas del sector La Lancha de Bucalemu, localidad perteneciente a la comunidad de Paredones en la Región del Libertador Bernardo O`Higgins. Los algueros son también llamados los temporeros del mar o mareros, debido a que habitan en las costas, solo en épocas de extracción.

Hombres y mujeres de estas familias fueron afectados por el tsunami provocado por el terremoto del 2010, en donde gran parte de sus viviendas temporales (rucos) en La Lancha desaparecieron, pero gracias a los conocimientos ancestrales sobre el medio ambiente natural, subieron a las partes más altas de Bucalemu, lo que posibilitó que no se produjeran pérdidas humanas. Pese a los riesgos y pérdidas materiales, reconstruyeron sus rucos en las mismas zonas de riesgos, lo que demuestra que pese a los desastres que han sufrido vuelven a estos lugares, mostrando de esta manera su dependencia y adaptación con el medio ambiente para subsistir, considerando que son ellos los que manejan con mayor experiencia los ciclos naturales de la renovación de recursos.

Desde esta perspectiva, la relevancia de esta investigación radica en que los conocimientos y experiencias propios de las comunidades pueden transformarse en soporte para las gestiones de carácter ambiental, especialmente aquellas con un objetivo de reducción de riesgos ${ }^{1}$. Además, servirían de insumos para la planificación del territorio.

1 Wilches-Chaux, 1998. 


\section{Metodología}

Metodológicamente se trabajó con un enfoque mixto, que es el más idóneo de acuerdo a las características de este estudio que según Hernández, Fernández y Baptista ${ }^{2}$, constituye el mayor nivel de integración entre los enfoques cualitativo y cuantitativo.

De lo cualitativo se propuso rescatar las experiencias que construyen los algueros en torno a su hábitat residencial, en tanto que desde el enfoque cuantitativo se obtuvo información de fuentes como el último censo, e investigaciones realizadas a los algueros mediante tesis de formación y otras investigaciones, todas ellas detalladas más adelante, sumado a la información arrojada en terreno por las familias entrevistadas. Esta información permitió medir ciertas características sociodemográficas y que servirán como insumo para establecer y definir futuras propuestas, partiendo de la base de la relación entre naturaleza y hombre.

Se utilizó una muestra de orden estructural, cada sujeto de la comunidad ve desde su particularidad y aporta información relevante, planteando una perspectiva propia y diferenciada que parte del colectivo. Se intentó representar una red de relaciones, de modo que cada participante pudo entenderse como una posición en una estructura.

Los criterios de selección de la muestra consistieron en:

- Cuatro familias pertenecientes a la comunidad de algueros de Bucalemu, sector La Lancha. Considerando que el universo de algueros de La Lancha es de 9 familias.

- A lo menos dos generaciones familiares dedicase a la extracción.

Ese fue el planteamiento con el que se partió, sin embargo las conversaciones grupales incluyeron al total de las familias de La Lancha. Las 9 familias tomadas como muestra de los 990 algueros de Cardenal Caro resulta un número acotado, debido a que lo que se pretende estudiar es la dinámica a nivel de "asentamiento", de esta manera se estudió el sentido de los comportamientos en torno al riesgo socio natural, y el significado de las prácticas en función de él. La muestra no necesariamente representó a todo el litoral, sino más bien fue un caso emblemático de resiliencia.

Hernández, Fernández y Batista, 2006. 


\section{Gestión del riesgo, una estrategia de prevención de desastres naturales}

Lo que han criticado diferentes investigadores -que se han enfocado en temas de desastres socionaturales-, frente a los manejos del riesgo, es la carencia de estudios de vulnerabilidad social pre desastres naturales, considerando que los eventos naturales se vuelven desastres siempre y cuando exista una sociedad frágil, expuesta y con falta de resiliencia (falta de organización, pobreza, etc.). En resumen, el riesgo socio-natural es la probabilidad de que una amenaza natural se transforme en desastre debido a la presencia de estos dos parámetros: una amenaza natural (por ejemplo un volcán) y un sistema social frágil ${ }^{3}$. Desde esta perspectiva, ha surgido como respuesta a estas inquietudes, la Gestión Integral del Riesgo a Desastres (GIRD), cuya estructura incorpora el análisis de las vulnerabilidades pre-existentes de un territorio ${ }^{4}$. Es decir, se concentra en la parte anterior y posterior a la probabilidad de ocurrencia de un desastre, con el fin de entregar herramientas para otorgar resiliencia y fortalecimiento a las dinámicas del territorio. En este sentido, incorporar la identificación de vulnerabilidades frente a amenazas naturales, es lo

3 Blaike, Cannon, David y Wisner, 1996.

4 Castro, 2005. que transformó a la gestión del riesgo en gestión integral del riesgo.

En el contexto rural, existen algunos autores como Simioni $i^{5}$ que afirman que estos territorios dependen de las zonas más urbanizados para sobreponerse a un desastre. Esta afirmación encuentra coherencia en el carácter menos avanzado con respecto a las zonas urbanas que se les ha asignado, generando un sesgo rural que contribuye a la calificación de territorios como urbanos solo por disponer de equipamiento público (hospitales, colegios, etc.), lo que no implica que todos sus habitantes tengan acceso a estos servicios, considerando las distancias alejadas en las que habitan algunas personas ${ }^{6}$.

En este escenario, en Chile, pese a existir una reconversión de los procesos de extracción o producción y aumento de otras actividades, la economía en algunos asentamientos rurales continúa dependiendo del medio ambiente natural ${ }^{7}$. Estas nuevas tendencias, en el caso de la Región de O’Higgins, han sido impulsadas desde el año 2010 por el Gobierno Regional, que pretende mejorar los niveles de producción agrícola por medio de la incorporación de proyectos y programas que aumentan las capacidades de negocios de la población y el

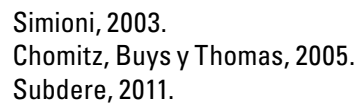


mejoramiento de instalaciones como el riego fis$\mathrm{cal}^{8}$. Sin embargo, aún falta incorporar la gestión del riesgo en la planificación del territorio, como lo explica el informe de Estrategia Regional de Desarrollo 2011-2020, realizado por el Gobierno Regional de O’Higgins. Si bien existen estudios del borde costero, estos aún no han sido publicados ${ }^{9}$ y los existentes no profundizan en las dinámicas propias de las comunidades.

En este sentido, el grado de vulnerabilidad de los territorios rurales o bien de asentamientos rurales que aún practican métodos artesanales en la extracción de recursos, tendrán calidad de vulnerable ante riesgos socio naturales, por su grado de ruralidad, dependencia con el cultivo, y nivel tecnológico ${ }^{10}$. se debe considerar además, su calidad de sistemas socio ecológicos cuya estructura está determinada por el vínculo con el medio ambiente.

El grupo de estudio de esta investigación se ubica en el secano interior y costero de Chile, que ha sido: "históricamente una zona de pobreza material"11. Ocupando una superficie del 30\% de la Región de O'Higgins, el secano de esta región es especialmente vulnerable, debido a la escasa capacidad de gestión, falta de organización de sus habitantes, altos índices de pobreza, alta dependencia

\footnotetext{
Soza y Meza, 2010.

Subdere, 2011.

Meza, Soza y Valle, 2011.

Soza y Meza, 2010, p. 35.
}

a la agricultura, escasez de agua, desempleo y falta de calificación de mano de obra. A esto se agrega que sus habitantes cultivan o extraen para su autoconsumo, comercializando solo sus excedentes ${ }^{12}$.

Por otro lado, cabe mencionar que es una de las zonas menos productivas de la región, debido principalmente a la escasez de agua y a la dependencia de la captación y utilización de aguas lluvia.

En el secano costero de la Provincia de Cardenal Caro se emplazan diferentes localidades ligadas al mar: Bucalemu, Topocalma, Matanzas y pequeños asentamientos temporales que se ubican en la costa, sumando un total de 990 algueros, de ellos 308 son mujeres y 682 hombres. Estos asentamiento no son identificados en ningún plano, su configuración en el borde mar se puede ver solo realizando un recorrido por ellos en los periodos de extracción, y todo el año para los casos de asentamientos permanentes.

De esta manera, a lo largo de la extensión nos podemos encontrar con las huellas dejadas por las comunidades (senderos u otros), con secados de algas y con los rucos que conforman los asentamientos ${ }^{13}$. De norte a sur se ubican Topocalma, El Hueso de Ballena, La Mala Bajá, Los Huachos, Las Quiscas, Los Leones, Punta Centinela, La Polcura,

12 Ibíd., 2010.

13 Araos, 2006. 
Carrizalillo, La Caceta, El Alto Colorado, El Agua Durán, Punta de Lobos, Punta La Sirena, La Mona, La Lancha, Las Cruces, La Quebradilla y Las Trancas. Los dos centros poblados que se relacionan con los asentamientos son Pichilemu y Bucalemu.

Los principales problemas ambientales del sector del secano costero de Cardenal Caro son las sequías y el aumento del turismo que ha degradado las fuentes de trabajo. Para el caso de estudio y en general para los asentamientos del borde ("orilla") del secano, las amenazas naturales a los que se ven expuestos -cuya actividad principal es la extracción de recursos marinos- van desde incendios forestales, marejadas, amenazas naturales a sus productos de extracción, y tsunamis post movimientos telúricos. Existen algunos problemas que no son de origen natural, que si bien nombraremos, no se profundizarán ya que no es el fin de este estudio- uno radica en que las nuevas generaciones están buscando otros tipos de trabajos, debido principalmente a que sus propios padres han fomentado el que continúen sus estudios en institutos o universidades, para dedicarse a otras labores que impliquen menos esfuerzo físico y menos sacrificios. Por otro lado, la alta demanda del producto podría provocar una sobre explotación del mismo.

En el 2010, parte del secano costero se vio afectado por un terremoto de grandes dimensiones que afectó a la zona central de Chile, especialmente las comunidades que viven de la extracción de productos del mar. Dentro de estas actividades las dos principales categorías corresponden a los recolectores de orilla y algueros, $(63,7 \%)^{14}$. Dentro de este porcentaje se encuentra el grupo objetivo de esta investigación, ubicado geográficamente al norte de la localidad de Bucalemu, perteneciente a la comuna de Paredones de la Región de O’Higgins y cuyo asentamiento es identificado bajo el nombre de La Lancha, cuya configuración en el borde mar se caracteriza por ser rural e informal, compuesto por nueve familias que se agrupan a orillas del mar, como ubicación estratégica para la extracción de algas en los meses de primavera/verano de cada año.

Pese a las amenazas naturales a los que están expuestos, los algueros se presentan como un grupo organizado, capaz de adaptarse a los cambios naturales que han experimentado. Esto, principalmente porque presentan un modo de vinculación con el medio ambiente que sigue cierto tipo de tradiciones, "en el que la dinámica de apropiación

14 SERNAPESCA, 2005. 
-tiempos y volúmenes extraídos- aún está determinada por los ciclos naturales de renovación de los recursos"15.

Esta actividad se ha mantenido durante más de 80 años en la región ${ }^{16}$. Por lo tanto, esta trayectoria demuestra conocimientos y experiencias en torno al borde costero, propios de la comunidad de algueros, que se caracterizan por ser trashumantes. La movilidad familiar, organizada de los algueros de Bucalemu- por el territorio costero, está determinada por el factor ambiental, ya que serán las temporadas secas las que propician el crecimiento de las algas, y la mejor habitabilidad de sus asentamientos ${ }^{17}$. En estas estaciones ellos viven en sus rucos $^{18}$ y terminada esta temporada, vuelven a sus casas hacia el interior de la comuna, distribuidas de manera dispersa en las partes altas de la localidad de Bucalemu. El hábitat de esta localidad se caracteriza por ser un centro poblado de categoría rural, en donde la concentración de población se enmarca entre las 1.001 a 2.000 habitantes, con un total de 1.435 personas $^{19}$. La localidad está

\footnotetext{
15 Morales, 2009, p. 66.

16 Pumarino, 2005.

17 Carvajal, Jofré, Girón, Leiva, Navarro, Quilaqueo y Silberman, 2010.

18 La denominación de ruco a las viviendas temporales en los asentamientos, es otorgada por los algueros del asentamiento La Lancha como los de toda la región de 0’Higgins. Cabe mencionar que su nombre alude a una vivienda precaria, que con el tiempo comenzaron a mejorar, y que actualmente se asemejan a viviendas básicas, a modo de medias aguas. inundaciones por ubicarse en zonas de riesgos.

Algunas condiciones en La Lancha, han mejorado, como por ejemplo, los accesos y sus rucos, sin embargo las formas de extraer siguen siendo las mismas, sin avances tecnológicos.

Los temporeros del mar han experimentado constantes procesos de adaptación, de los que se desprenden tradiciones con respecto al medio ambiente propias y que toman una connotación ancestral ya que: "el conocimiento aprendido en la orilla, ha constituido también la herencia más importante que hombres y mujeres del mar han podido entregar a sus descendientes" ${ }^{20}$. Y en ese sentido, las diferentes adaptaciones a los cambios de la naturaleza que este tipo de familias enfrentan, configuran una dinámica del hábitat residencial rural singular, en la que el riesgo socio natural es parte de su cotidianidad. De esta manera, desde esta condición, se desprenderán conocimientos, experiencias y saberes propios de su relación con la amenaza natural más

20 Carvajal, Jofré, Girón, Leiva, Navarro, Quilaqueo y Silberman, 2010, p. 48. 
evidente: "los comportamientos del mar" (tsunami o marejadas).

Estos conocimientos para el terremoto del 2010 cobraron importancia, ya que dirigidos por ellos, la población de algueros y bucaleminos subieron a los cerros evadiendo al tsunami provocado por el movimiento telúrico, lo que posibilitó que pese a que se perdieron sus rucos y los procesos de secados de algas, no ocurrieron muertes humanas.

El caso de este estudio se diferencia del resto de algueros distribuidos en el país, por haber estado expuestos a un desastre natural y resultar "vencedores" del contratiempo vivido. Lo que a primera impresión se muestra como una comunidad fuerte, capaz de enfrentar este tipo de eventos, que se muestra autosustentable y que no acostumbra a depender de la ayuda externa para sobreponerse. Dicho de otra manera, es una comunidad que ha practicado la gestión del riesgo, de manera espontánea, y en esto hay una connotación histórica con respecto a los usos del suelo y sus recursos, que ha contribuido a que su ordenamiento territorial se relacione precisamente con estos cambios. Si en un comienzo la comunidad trabajaba la tierra, luego con la forestación, la comunidad se vuelca hacia la orilla del mar, donde hay productos gratuitos a extraer.

Pese a que el oficio de alguero en el asentamiento de La Lancha podría desaparecer, -debido a que las nuevas generaciones están buscando nuevas formas de subsistir-, resulta interesante estudiar este caso; primero porque representa una realidad de 990 algueros en la región de O’Higgins, y segundo y no menos importante, por la relación con el patrimonio inmaterial que significa este tipo de comunidades, y esto tiene que ver con su identidad, sus costumbres y vivencias en la orilla. Un alguero o marero"21: "se reconoce como tal cuando está en el ruco con sus amigos y familiares, en plena temporada de recolección, pero es también hombre de mar cuando se aleja de la provincia"22. Esto, porque la identidad surge con las prácticas sociales desarrolladas en determinados territorios que han sido apropiados durante años. Y en ese sentido, diversas investigaciones, como las de Carvajal23 ${ }^{23}$ profundizan con una mirada histórica la itinerancia de los algueros de Bucalemu hasta hoy. Araos ${ }^{24}$ hace un recorrido por las costumbres de las comunidades de algueros, de todo el borde de Cardenal Caro; también

21 Recordemos que también se denominan mareros a los algueros. 22 Araos, 2006.p. 167.

23 Carvajal, Jofré, Girón, Leiva, Navarro, Quilaqueo y Silberman, 2010.

24 Araos, 2006. 
existen libros más recientes como el realizado por Cedesus ${ }^{25}$ y el equipo de Collofe Creativa en conjunto con el Centro de Desarrollo Sustentable de Pichilemu, cuyo objetivo es dar conocer el patrimonio inmaterial de las dinámicas de los algueros en el Borde Costero de la Provincia de Cardenal Caro. Si bien esta investigación no se focaliza en el patrimonio intangible, es importante destacarlo para, de algún modo, comprender la importancia de trabajar la relación de sus dinámicas con los riesgos socio-naturales a los que están expuestos.

Por otro lado, en Cardenal Caro la producción de esta zona está determinada por la extracción de algas, alcanzando por ejemplo ya en el año 2004 las 2.262 toneladas de desembarque de un total de 3.201 de los productos costeros, según datos del Servicio Nacional de Pesca ${ }^{26}$. A nivel nacional, según el Instituto de Fomento Pesquero (IFOP), en el 2010 se exportaron 74.095 toneladas de algas y sus derivados, lo que significa retornos de 183 millones de dólares.

Cabe destacar que la demanda de algas ha ido en aumento, tal como lo identifican los algueros en esta investigación. Además las mismas organizaciones

25 Centro de Desarrollo Sustentable, 2011.

26 SERNAPESCA, 2005. locales -como afirma Araos $^{27}$ - reconocen este oficio como importante incluso a nivel nacional.

En el caso de La Lancha, luego del tsunami del 2010 se perdieron todos los rucos y posteriormente, con ayuda de organizaciones sin fines de lucro, ellos volvieron a construir en las mismas zonas de riesgo, adaptándose a los cambios sufridos por el desastre, lo que evidencia dependencia a los recursos del borde. Este vínculo con los recursos se puede comprobar, por ejemplo, en la investigación de $\operatorname{Araos}^{28}$ en donde concluye que reconoció un sistema de economía local basada en la compresión y explotación total de los recursos de la orilla. En ese sentido, tal como lo explica el autor, esto se divide por un lado en "sobrevivencia familiar", es decir, desde lo cotidiano que implica mantenerse en los rucos: el huerto familiar, las gallinas, la caza de pájaros, la recolección de mariscos y pescas para la alimentación. Y por otro lado, la producción comercial, que es la que fundamenta su "dependencia a las algas", como oficio preponderante en sus vidas. Ambos aspectos de cotidianidad y de producción formarían su economía local.

Lo anterior, sumado a las tradiciones que conforman su identidad, explicaría la persistencia de volver a los mismos lugares a recolectar y emplazar

27 Araos, 2006.

28 Ibíd. 
sus rucos. Además de los riesgos naturales a los que están expuestos, también encuentran otro tipo de barreras con las que deben lidiar, y que tienen que ver con los conflictos históricos que han existido con los dueños de los fundos y que se mantienen hasta el día de hoy ${ }^{29}$. Esto debido a que los predios alcanzan áreas del borde costero, produciéndose una ambigüedad en los límites de los terrenos, lo que ha provocado conflictos con los orilleros, pese a que la Ley de Pesca y Acuicultura de 1991 permite la utilización de estas áreas. Sin embargo, actualmente los conflictos si bien han disminuido, estos continúan.

En el escenario de estudios de vulnerabilidades frente a los riesgos socio naturales, las gestiones en algunas ocasiones no consideran las riquezas y conocimientos que tienen las comunidades de su entorno. El considerarlas por medio de su conocimiento, análisis, y difusión pueden transformarse en soporte para las gestiones de carácter ambiental, especialmente aquellas con un objetivo de reducción de riesgos ${ }^{30}$. De este modo, pueden estas gestiones tornarse más eficientes y evitar la generación de nuevos escenarios de riesgos o bien evitar incongruencias con la realidad local, además de aprovechar las potencialidades propias de la comunidad.

29 Carvajal, Jofré, Girón, Leiva, Navarro, Quilaqueo y Silberman, 2010.

30

Wilches-Chaux, 1998.
Desde esa perspectiva este artículo, como parte de la investigación llevada a cabo, pretende comprender las experiencias, características y conocimientos en torno al manejo de recursos naturales en escenarios de riesgos, surgidas de las dinámicas del hábitat residencial rural del borde mar, propio de una comunidad como la de los algueros del secano costero, y que puedan adaptarse o incluirse en las gestiones públicas y privadas enfocadas en prevenir y enfrentar desastres socio-naturales, desde el punto de vista de considerarlas, respetarlas y potenciar aquellos aspectos que se presentan fuertes o bien intervenir en los elementos débiles de la estructura de este tipo de sistemas socio-ecológicos.

Frente a este panorama surge la siguiente pregunta: ¿cómo se adaptan los algueros de La Lancha de Bucalemu, al riesgo socio-natural al que están expuestos? Para responder esta pregunta, se estableció como objetivo general la importancia de analizar la adaptación al riesgo socio-natural que tienen los extractores de algas de la localidad de La Lancha en Bucalemu, con el fin de contribuir desde una mirada holística a las medidas de mitigación del riesgo socio-natural.

Desde esa perspectiva, surgieron los siguientes objetivos específicos: 
- Identificar la configuración del riesgo socio natural de los algueros de La Lancha.

- Identificar cómo se han adaptado las dinámicas de los algueros de La Lancha en relación al riesgo socio natural al que se ven expuestos.

Como hipótesis se planteó que la comunidad de algueros de Bucalemu, ha configurado una gestión del riesgo espontánea, producto de su constante adaptación a los cambios naturales en el territorio.

\section{Dinámicas del hábitat residencial rural}

En la mayoría de los casos, el enfoque territorial institucionaliza "espacios" que por lo general ya han sido apropiados por las comunidades ${ }^{31}$. Desde esta perspectiva, en el caso rural la apropiación de lugares por parte de sus habitantes está fuertemente vinculada con la dependencia a la extracción y manejo de recursos naturales. De esta manera, se puede observar que la ubicación de sus asentamientos está condicionada en parte por costo y beneficio en relación con sus lugares de trabajo, específicamente con sus actividades de producción.

31 Echeverri y Sotomayor, 2010.
Por lo general, esta dependencia construye el hábitat de las zonas rurales a pesar de los riesgos que se corren.

Como ya lo comentamos antes, tradicionalmente a los territorios rurales se les ha dado un carácter de marginal al calificarlos como atrasados en comparación con las zonas urbanas. Basándose en esto último, es que se clasifica a ciertos territorios como rurales por el solo hecho de no disponer de servicios básicos ${ }^{32}$. De este modo, ciertos lugares son calificados urbanos por disponer de hospitales, escuelas y carreteras pavimentadas. Esta manera de enmarcar la ruralidad puede contribuir a una antiruralidad que dificulta la realización de políticas públicas adecuadas, debido a que la existencia de servicios básicos no garantiza que todos sus habitantes tengan acceso a ellos, sobre todo considerando el aislamiento geográfico en que se encuentran ciertos asentamientos.

El tema de ruralidad es amplio, existen grados y tipos, como lo plantea Berdegué y otros investigadores $^{33}$. Incluso la definición de lo que es ruralidad en las políticas públicas de América está definida por el tamaño poblacional, y en Chile la definen además por la actividad preponderante y la importancia a nivel económico. Así, Berdegués ${ }^{34}$ definió 9 tipos de comunas rurales; i) un $44 \%$ de la pobla-

32 Chomitz, Buys y Thomas, 2005.

33 Berdegué, Jara, Modrego, San Clemente y Schejtman, 2010.

34 Ibíd. 
ción rural se ubica en comunas con un grado de ruralidad intermedia, ii) comunas con alta dependencia a la agricultura y aislados geográficamente, iii) comunas dependientes de la agricultura, pero con ruralidad intermedia, iv) comunas en donde el turismo cobra importancia, v) comunas con base económica pesquera o acuícola, y por último vi) comunas rurales cercanas a un polo urbano-rural.

El caso de estudio se ubica en la localidad de Bucalemu, perteneciente administrativamente a la comuna de Paredones, definida por Berdegué ${ }^{35}$ como fuertemente rural.

Para efectos de esta investigación se tomaron los siguientes fundamentos teóricos que se encauzan hacia una definición de la nueva ruralidad, tomando en cuenta que con el concepto de tipo de ruralidad "se da cuenta de las diferencias en las configuraciones socioeconómicas de cada territorio"36.

a) La dependencia económica con el medio ambiente contribuye a la formación de atributos que surgen de los recursos naturales; vale decir, las dinámicas que se estructuran en torno a las actividades económicas, redes y procesos de formación social ${ }^{37}$.

35 Ibíd.

36 Ibíd, p. 5.

37 Sepulveda, Rodríguez, Echeverri y Portilla, 2003. b) Una visión económica con un enfoque básico, en donde se afirma que la formación de asentamientos humanos esta ligada a la localización de su base económica. Sin embargo, en el caso rural el factor que lo diferencia de lo urbano es la importancia de la renovación y oferta de los recursos naturales: "determina patrones de apropiación y permanencia en el territorio en procesos históricos" 38 .

c) El espacio rural visto desde una mirada que ya no toma como actividad preponderante a la agricultura, sino que permite incluir más actividades que esta, como la pesca, turismo, etc. ${ }^{39}$

d) Y por último, un enfoque que permite considerar que los territorios rurales pueden contener centros urbanos que dependan de la utilización de los recursos (turismo, pesca, deporte, agricultura, etc.), cuando el límite entre lo urbano y rural se torna difuso ${ }^{40}$.

Este último punto en cuanto a agricultura y acuicultura, pese a que su participación se ha visto disminuida en el territorio rural, sigue siendo la directriz que los define, por el tipo de economía localizada, que está vinculada a la ubicación y oferta de recursos naturales disponibles ${ }^{41}$.

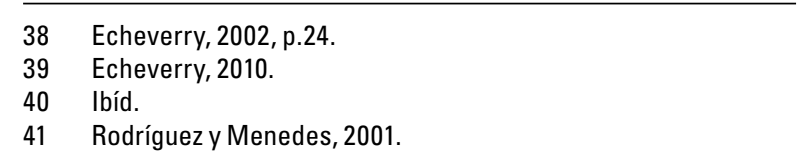


Desde esta perspectiva, el enfoque de esta investigación tiene relación con las dinámicas que se generan entre residencias y lugares de trabajo (extracción de algas), adquiriendo entonces un carácter temporal en relación a cómo la comunidad se va adaptando a las condiciones naturales del territorio para la extracción de sus productos. Como lo plantea Morales ${ }^{42}$, en el caso de los algueros de Pichilemu, es una dinámica entre tiempos y volúmenes extraídos, dependientes de las renovaciones de los recursos.

Los asentamientos de algueros de toda la extensión del secano costero de Cardenal Caro, se dividen en aquellos que son permanentes, en el sentido de que viven ahí todo el año, sin una segunda vivienda y otros del tipo semipermanente por habitarlos solo en épocas de extracción. Se caracterizan por ser de precarias condiciones, situación que ha ido evolucionando desde los inicios, en que se adaptaban a los refugios naturales del lugar, hasta ahora que se organizan en rucos autoconstruidos. Los rucos son viviendas cuya distribución interior se asimila a un caserío campesino, y que forman en su conjunto pequeños asentamientos ${ }^{43}$. Organizados en el lugar por sus lazos familiares, se han manteni-

42 Morales, 2009.

43 Araos, 2006.

158 revista invi 32(89) : 145-169, mayo 2017 do por años. A la fecha, según las conversaciones sostenidas, no recuerdan cómo han elegido cada uno su ubicación.

Masuda ${ }^{44}$, con respecto a las costas chilenas, clasifica a los pueblos recolectores en: i) asentamientos de estilo de vida simple, en donde los recolectores se refugian por dos o tres meses, en cuevas o en salientes de las rocas y que trabajan de manera individual, y que carecen de todo tipo de organización, solo en algunas ocasiones tienen algún líder, pero que no ejerce liderazgo más allá del asentamiento, quedando de esta manera la comunidad a merced de intermediarios; ii) el segundo tipo, se refiere a una congregación de chozas de paja, que se agrupan a modo de aldeas, con características sociales similares al primer grupo; iii) el tercer grupo, corresponde a asentamientos sedentarios, con viviendas de adobe, en donde siempre hay un líder con características de empresario.

Las convivencias surgidas producto del trabajo en el medio ambiente natural por años, propician entonces un ordenamiento territorial histórico ${ }^{45}$. Esta visión, la de abordar la espacialidad y for-

\section{Masuda, 1986}

45 La mirada acerca del ordenamiento territorial histórico y en general de la apropiación del espacio como resultado de procesos históricos, hace referencia a la manera espontánea de ocupary usar el territorio. 


\section{FIGURA 1. DISTRIBUCIÓN RUCOS}

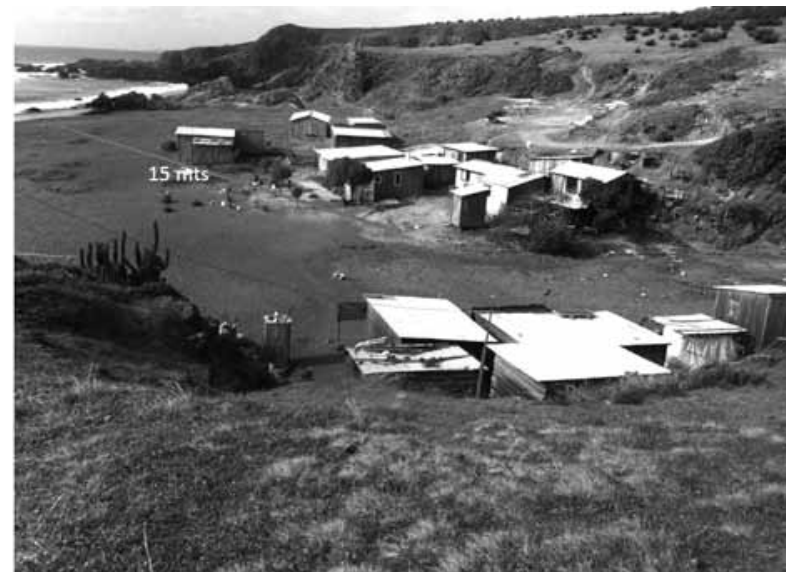

Fuente: Elaboración propia, 2014.

mación de un territorio, contribuye a entender los mecanismos de producción del espacio, porque muestra las interacciones sociales con respecto a la apropiación y distribución de los excedentes que pueda propiciar la naturaleza.

\section{FIGURA 2. RUCO}

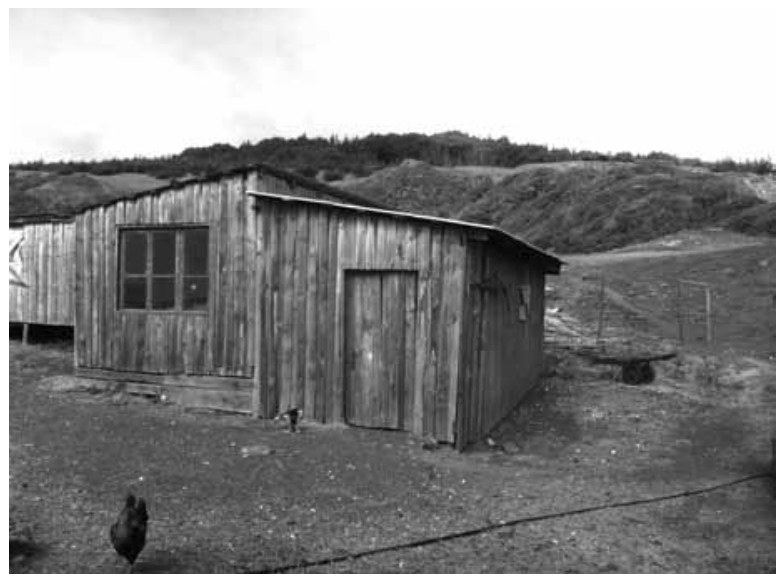

Fuente: Elaboración propia, 2014.

\section{Hacia una gestión adaptativa de los Sistemas Socio Ecológicos (SSEs)}

El concepto de Sistemas Socio-Ecológicos (SSEs), se utiliza con el fin de involucrar todas las dimensiones del hábitat residencial. Desde este punto de vista es un concepto holístico, que pretende integrar ser humano y naturaleza, pues dialogan diferentes escalas del hábitat (naturaleza, individuos, política, economía y aspectos temporales).

Los seres vivos nos adaptamos a los cambios y en ese proceso podemos transformar el medio ambiente como consecuencia de nuestras propias necesidades. 
Por ejemplo, Walker ${ }^{46}$ muestra la situación en dehesas, en donde el sistema social está condicionado económicamente por la cantidad existente de césped, arbustos y ganado. Sin embargo, se pueden introducir nuevos "paisajes estables" o más bien nuevas formas de ganarse la vida, como por ejemplo el ecoturismo, basado en los atributos del lugar (ríos, bosques y fauna). De esta manera, el término adaptativo se relaciona con la capacidad de prosperar cuando hay un cambio.

El enfoque de esta investigación con respecto a la gestión del riesgo se asoció a los "manejos adaptativos" a los cambios, tanto lentos como violentos de la naturaleza. Y adquiere relevancia, en la medida en que dicha gestión, considere los siguientes conceptos: resiliencia, adaptabilidad y transformabilidad.

Según Walker ${ }^{47}$ se definirían de las siguientes maneras:

a) Resiliencia es la capacidad de un sistema de absorber los disturbios y reorganizarse mientras se lleva a cabo un cambio y aun así retener esencialmente la misma función, estructura, identidad y feedback.

46 Walker, Holling, Carpenter y Kizing, 2004

47 Ibíd. b) Adaptabilidad es la capacidad de los actores humanos de un sistema de manejar intencionalmente o no la resiliencia.

c) Transformabilidad es la capacidad de crear nuevos "paisajes estables" o nuevos sistemas cuando las condiciones económicas, sociales, políticas o ecológicas hacen que la existencia no se pueda sostener. De este modo, significa crear nuevos paisajes estables, incorporando nuevas maneras de "ganarse la vida", cambiando variables que definen el sistema.

Por otra parte, los sistemas socio ecológico desde el punto de vista de Holling ${ }^{48}$ se puede decir que no son tan impredecibles frente a este tipo de eventos, ya que presentan un ciclo adaptativo que se configura por: crecimiento, acumulación, reestructuración y renovación. Identificar estos ciclos nos puede servir para fortalecer la resiliencia ${ }^{49}$, debido a que mientras suceden las fases del ciclo -si ocurriese algún evento- dependerá de este aspecto para saber la rapidez y forma de recuperación que tenga el sistema. Por tanto habrá que entender las probabilidades de recuperarse en cada una de estas etapas imaginando un escenario de crisis en cada fase.

48 Holling, 2001.

49 "Es un proxy de la habilidad del sistema para reorganizarse como reacción ante los cambios en el entorno, su capacidad de aprendizaje, su flexibilidad para experimentary adoptar soluciones innovadoras, así como para desarrollar una multiplicidad de respuestas ". (Albalá, 2007, p. 32; Walker, Holling, Carpenter y Kizing, 2002). 


\section{FIGURA 3. ESOUEMA SISTEMA SOCIO ECOLOGICO.}

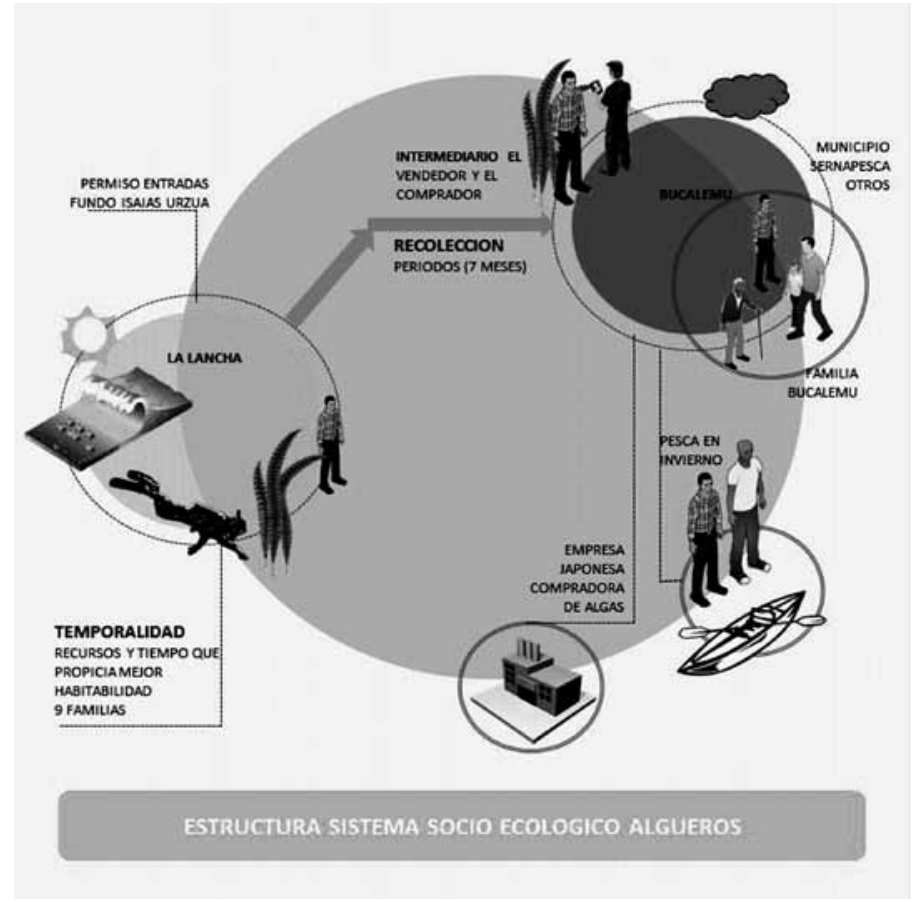

Fuente: Elaboración propia, 2014.

Recordemos que los ciclos adaptativos son una condición del sistema, que trae como consecuencia que los sistemas sean dinámicos. En ese contexto el ciclo adaptativo de los algueros de La Lancha, está condicionado a los factores del clima, y este a su vez con las estaciones verano-invierno. Por lo tanto, el ciclo desde la interpretación de esta investigación es anual, ya que cada año existen las etapas de crecimiento, acumulación, reestructuración y renovación condicionada a la extracción de recursos, ganancias económicas, y estructura del sistema socio ecológico, para dar pie en cada 
temporada a una nueva renovación, tanto de recursos como de organización, para obtenerlos.

Los elementos principales que componen el sistema socio ecológico de los algueros son: a) nueve familias, b) un intermediario que comercializa el producto, c) un comprador, que también puede ser una fábrica como la actual existente, de origen japonés (instalada hace algunos años en Paredones), e) algas y medio ambiente marino, f) hábitat de Bucalemu y hábitat de La Lancha.

\section{Vulnerabilidad de los territorios rurales frente a los riesgos socio-naturales}

El espacio en donde se desarrolla la actividad de los algueros configura su estructura a partir de los asentamientos temporales o permanentes en el territorio, considerando que existen algunos asentamientos como Topocalma, donde habitan todo el año en la orilla. El proceso de apropiación corresponde a terrenos marginales, en el límite de los fundos; al sur el fundo Tanumé y al norte el fundo Las Cruces. Las familias comenzaron a instalarse en lugares donde la competencia por los recursos fuera menor ${ }^{50}$.

La diferencia entre pescadores y algueros desde el punto de vista del hábitat radica en los elementos usados del territorio que tienen. Así lo define Araos $^{51}$, en el estudio de los algueros de Cardenal Caro; él dice que en el caso de los pescadores nos referimos a la caleta, al bote y altamar, y en el caso de los algueros hablamos de la orilla en donde se reconocen los roqueríos, la playa, los cerros y el mar.

En este escenario dependientes de las actividades económicas, generadas en el medio ambiente natural-, la vulnerabilidad socio-natural frente a los cambios tanto abruptos como lentos que se producen en el medio ambiente (terremotos, maremotos, sequias, etc.), adquieren relevancia. Esta se genera, primero, por el estado de los elementos de un territorio según las variables de: fragilidad, falta de resiliencia y exposición, explicado a continuación por medios de los efectos sobre las viviendas luego de lluvias intensas ${ }^{52}$ :

- Sensibilidad, las viviendas más precarias sufrirán mayores daños por inundaciones.

50 Araos, 2006

51 Ibíd.

52 Un tema de desarrollo (...), 2000. 
- Exposición, la ubicación de viviendas en sectores con riesgo de inundaciones tendrán mayores efectos negativos.

- Capacidad de respuesta, los recursos disponibles de las familias que sufrieron daños en sus viviendas condicionarán la capacidad de reconstruir lo dañado.

Estos se traducen en el caso rural por las siguientes variables $^{53}$ :

- Grado de ruralidad: Lo podemos identificar apoyándonos en el seguimiento de parámetros de lo más urbano a lo más rural, con el apoyo de los datos censale, y en la distribución de la población (a mayor distribución de la población en el territorio mayor grado de ruralidad) ${ }^{54}$.

- Nivel tecnológico: Desde los aspectos sociales los rubros con mayor tecnología y capital son menos vulnerables ante los cambios en la producción que aquellos rubros más simples, los cuales están asociados a propietarios con menos recursos económicos ${ }^{55}$.

- Nivel de dependencia económica del cultivo: El factor UCT (uso de capital y tecnología) se construye en base al nivel de insumos y tecnologías utilizados por cada rubro productivo.

\footnotetext{
53 Meza, Soza y Valle, 2011.

54 Berdegué et al, 2010.

55 Meza, Soza y Valle, 2011.
}

Este criterio es para ver la cantidad de personas que trabajan en un territorio productivo, la cantidad de recursos para mantención, maquinaria, pesticidas, fertilizantes.

Se explican las relaciones en la figura 4 .

Así, el riesgo de desastre socio-natural se estructura por dos componentes: uno físico, que constituye la amenaza natural, como probabilidades de cambios naturales que puedan ser factor de desastre en un determinado territorio. Y otro elemento es el social, que se compone por la capacidad de respuesta, tanto desde el punto de vista de respuesta de las personas como de las condiciones que estas tienen en términos materiales, que presentándose frágiles estarían conformando el riesgo socio natural.

\section{El riesgo socio natural}

Según la figura 4, se puede observar cómo se va configurando el riesgo socio natural en un territorio rural, partiendo de la base de que se define como la probabilidad de que una amenaza natural frente a un grupo vulnerable se transforme en desastre, desde ese principio decimos que un asentamiento puede estar en riesgo socio natural.

El fundamento para utilizar este concepto se basó en la siguiente definición: 


\section{FIGURA 4. CONFIGURACIÓN RIESGO SOCIO NATURAL EN ZONAS RURALES}

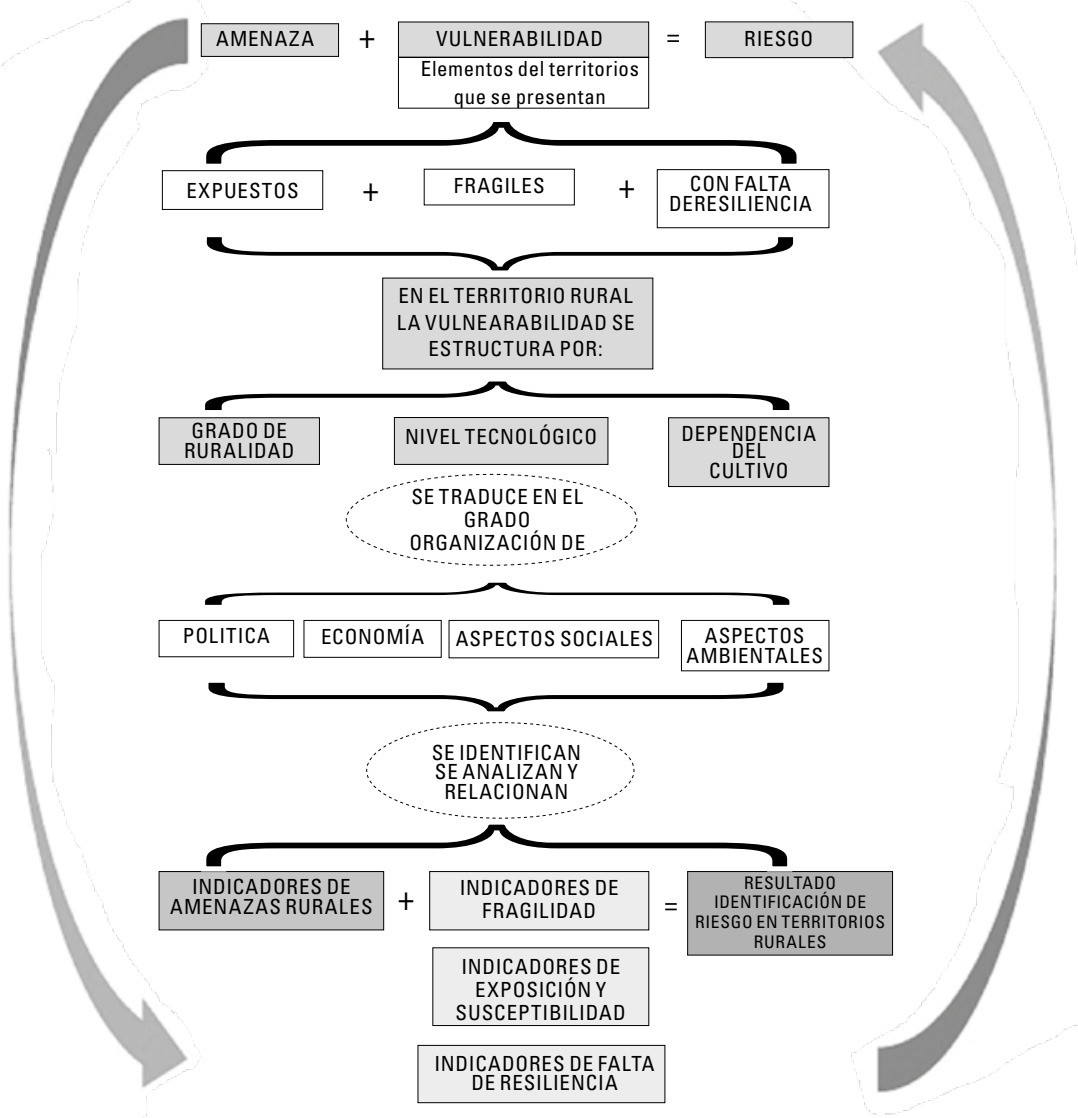

Fuente: Elaboración propia. 


\begin{tabular}{ll}
\hline Sistema complejo & Sistema socio ecológico \\
\hline Potencial & Recursos, infraestructura, recursos humanos. \\
\hline Control Interno & Enraizamiento-vinculación, gobernanza. \\
Resiliencia & $\begin{array}{l}\text { Vulnerabilidad, dependencia de recursos naturales, integración en mercados, diversifica- } \\
\text { ción de renta. }\end{array}$
\end{tabular}

Fuente: Albalá, 2007.

"El riesgo es el resultado de la interacción, en el tiempo y el espacio, de los eventos físicos potenciales y los elementos expuestos y vulnerables de los sistemas sociales y ambientales. En tal interacción, esos eventos físicos se transforman en amenazas con verdadero potencial de generar futuras pérdidas y daños" 56 .

En ese contexto es que la importancia de identificar el riesgo, radica en que con esto podemos construir una gestión del riesgo adecuada para una realidad sin tener que generalizar para todos los casos.

El riesgo socio natural es un requisito de futuros desastres, que pueden ser de pequeña o gran escala.

Cardona, Bertoni, Gubbs, Hermilin y Lavell, 2010, p.17.

\section{El riesgo socio natural de los algueros de Bucalemu, desde su configuración hacia su adaptación.}

Las características de un sistema socio ecológico complejo están guiadas por los conceptos de: potencial, control interno y resiliencia. Y estas a su vez están divididas por una serie de variables que ayudan a comprender estos sistemas.

En el recorrido de esta investigación se revisó cómo los algueros de La Lancha se han ido "acomodando" o adaptando a diferentes situaciones en relación con su medio ambiente, con o sin perturbaciones externas que puedan afectar su estructura de relaciones entre recursos, medio ambiente 


\section{TABLA 2. SISTEMA SOCIO ECOLÓGICO DE LOS ALGUEROS DE LA LANCHA}

\begin{tabular}{|c|c|c|c|c|c|}
\hline & & Vulnerabilidad & & otros & \\
\hline & Dependencia al cultivo & Nivel tecnológico & Grado de ruralidad & $\begin{array}{l}\text { Integración en } \\
\text { mercados }\end{array}$ & $\begin{array}{l}\text { Diversificación } \\
\text { de renta }\end{array}$ \\
\hline $\begin{array}{l}\text { Sistema } \\
\text { complejo }\end{array}$ & $\begin{array}{l}\text { Alta dependencia } \\
\text { a los recursos na- } \\
\text { turales, pese a que } \\
\text { pueden tener trabajos } \\
\text { alternativos. }\end{array}$ & $\begin{array}{l}\text { Bajo nivel tecnoló- } \\
\text { gico en sus modos } \\
\text { de extracción, ya } \\
\text { que utilizan herra- } \\
\text { mientas artesana- } \\
\text { les la extracción. }\end{array}$ & $\begin{array}{l}\text { Localidad de Buca- } \\
\text { lemu con alto grado } \\
\text { rural (Berdegué). }\end{array}$ & $\begin{array}{l}\text { Desde hace unos } 5 \\
\text { años se han integrado } \\
\text { nuevas demandas de } \\
\text { productos, así mismo } \\
\text { venden a nuevas } \\
\text { empresas extranjeras } \\
\text { que se han instalado } \\
\text { en el sector. }\end{array}$ & No aplica. \\
\hline & & $\begin{array}{l}\text { Nivel alto en tecno- } \\
\text { logías con respecto } \\
\text { a las comunicacio- } \\
\text { nes en La Lancha } \\
\text { (celulares, PC, Tv, e } \\
\text { internet). }\end{array}$ & $\begin{array}{l}\text { Capacidad de autor- } \\
\text { ganizarse luego de } \\
\text { alguna perturbación. }\end{array}$ & & \\
\hline
\end{tabular}

Fuente. Elaboración propia.

y dinámicas sociales. Algunos de sus elementos, como por ejemplo su cohesión social y sus conocimientos, se presentan fuertes, mientras que otras se muestran extremadamente débiles, como por ejemplo, la ubicación y calidad de los rucos de La Lancha, la carencia de organismos públicos que velen por su seguridad o la distancia a los centros urbanos, entre otros.
Por otro lado, recordemos que la resiliencia es la capacidad de un sistema socio ecológico de enfrentar una perturbación que afecte a su estructura, manteniendo su misma función, estructura e identidad. En este sentido se diagrama la relación de resultados en la tabla2.

Uno de los conceptos más trascendentales con respecto a la resiliencia tiene relación con la capacidad de autoorganización de los sistemas socios 
ecológicos y cómo esto influye en la gobernanza del sistema ${ }^{57}$.

\section{Conclusiones}

En el caso de los algueros, históricamente la autoorganización va ligada a sus lazos familiares y a su énfasis en trabajar libremente, situación que los ha obligado a defenderse ante la adversidad. Esto se ve reflejado en su forma de organización en cada temporada y en cómo levantaron sus asentamientos luego del tsunami, pese a que fueron ayudados por algunas organizaciones externas.

Una vez identificado el riesgo socio natural que afecta a los algueros de La Lancha, la fase siguiente de la investigación contempla evaluar alternativas que permitan desarrollar una gestión integral del riesgo de desastres socionaturales. A esto se le incorporará una gestión adaptativa de los sistemas socioecológicos.

La importancia de proyectar escenarios de riesgos con el fin prever posibles desastres para su preparación, contribuye directamente a fortalecer la resiliencia. En el caso estudiado, se rescata que para que se desarme la estructura del sistema de los algueros, tendría que ocurrir eventos que no solo dañen al asentamiento en el plano físico-espacial, sino además al producto estructurante que son las algas. Y es en ese sentido, donde se deben buscar alternativas, ya sea de protección en cada casa de eventos, de mitigación o bien de trabajos alternativos para la comunidad, potenciar esto último sería una estrategia de resiliencia.

Una meta importante o reto que se puede plantear frente a estos territorios, consiste en su desmarginalización. Para ello se deberá tener en cuenta: i) acuerdos con la comunidad consensuados, ii) integrar experiencias previas de todos los agentes involucrados, iii) seguros a la extracción.

Por último y de gran importancia es el aspecto del ordenamiento territorial, esto en relación con los derechos del suelo de asentamientos como el de los algueros, debido a los conflictos causados por la falta de acceso a la orilla y tener mayor protección en lugares más altos. Desde esta evidencia se hace necesario considerar que el ordenamiento territorial es una buena herramienta para la gestión del riesgo, en el sentido de incorporar las actividades humanas.

De alguna manera, más que definir las estrategias para mitigar los riesgos o armar una política de gestión del riesgo, es más que nada "volver atrás" en el sentido de poder dimensionar los fenómenos naturales e incorporarlos y convivir con ellos tal como hacían nuestros antepasados, es decir, conseguir dialogar con la naturaleza.

57 Abel, Cumming y Anderies, 2006. 


\section{Bibliografía}

ABEL, Nick; CUMMING, David H. M. y ANDERIES, John M. Collapse and reorganization in social-ecological systems: questions, some ideas and policy implications. Ecology and Society. 11(1), 2006. ISSN 1708-3087.

ALBALA, Mateo. Elementos institucionales en las zonas rurales: una propuesta metodológica para su identificación y valoración en comarcas de Andalucía y Nicaragua. Córdoba, Facultad de Ciencias Económicas y Empresariales, ETEA, Universidad de Córdoba. 2007. Tesis doctoral.

ARAOS, Francisco. Irse a la orilla. Una aproximación etnográfica a los Mareros de Cardenal Caro. Universidad de Chile. 2006. Memoria para optar al título de antropólogo.

BERDEGUÉ, Julio; JARA Esteban; MODREGO, Félix; SANCLEMENTE, Ximena y SCHEJTMAN, Alexander. Comunas rurales de Chile. Santiago, Rimisp Centro Latinoamericano para el Desarrollo Rural. 2010.

BLAIKIE, Piers; CANNON, Terry; DAVID, Ian y WISNER, Ben. Vulnerabilidad. El entorno social, político y económico de los desastres. La Red, Red de Estudios Sociales en Prevención de Desastres en América Latina. 1996.

CARDONA, Omar; BERTONI, Juan Carlos; GUBSS, Tony; HERMILIN, Michel y LAVELL, Allan. Entendimiento y gestión del Riesgo asociado a las amenazas naturales: un enfoque científico integral para América Latina y el Caribe. Río de Janeiro. ICSU, LAC / CONACYT, 2010. ISBN 978-0-930357-77-1.

CARVAJAL, Daniel; JOFRÉ, Rodrigo; GIRÓN, Nicolás; LEIVA, Marianela; NAVARRO, Jorge; OUILAQUEO Víctor y SILBERMAN Analía. Entre el campo y el mar, cosechando la orilla. El tránsito histórico de los trabajadores itinerantes de Bucalemu en el siglo XX. Santiago, TLAHC. 2010.

CASTRO, Cecilia. La inequidad de género en la gestión integral del riesgo de desastre. Un acercamiento. Revista de la Universidad Cristóbal Colón. (20), 2005.

CENTRO de Desarrollo Sustentable. Cambio climático y recursos hídricos: escenarios, impactos y medidas de adaptación. CEDESUS. 2011. Disponible en: http://cedesus.blogspot.cl/2011/10/cambioclimatico-y-recursos-hidricos.html.

CHOMITZ, Kenneth M., ed.; BUYS, Piet, ed. y THOMAS, Timothy S., ed. Quantifying the rural-urban gradient in Latin America and the Caribbean. World Bank Policy Research Working Paper. (3634), junio 2005. DOI 10.1596/1813-9450-3634.

ECHEVERRI, Rafael y RIBER0, María Pilar. Nueva ruralidad. Visión del territorio en América Latina y el Caribe. San José, Costa Rica: Instituto Interamericano de Cooperación para la Agricultura IICA. 2002.

ECHEVERRY, Rafael y SOTOMAYOR, Octavio. Estrategias de gestión territorial rural en las políticas 
públicas en Iberoamérica. Santiago de Chile, CEPAL. 2010

HERNÁNDEZ SAMPIERI, Roberto; FERNÁNDEZ-COLLADO, Carlos y BAPTISTA LUCIO, Pilar. Metodología de la investigación. México, Mc Graw-Hill Interamericana. 2006. ISBN 970-10-5753-8.

HOLLING, Crawford Stanley. Understanding the complexity of economic, ecological, and social systems. Ecosystems. 4(5): 390-405, 2001. DOI 10.1007/s10021-001-0101-5.

MASUDA, Shozo. Las algas en la etnografía andina de ayer y hoy. En: MASUDA, Shozo. Etnografía e historia del mundo andino. Continuidad y cambio. Tokio, Universidad de Tokio. 1986.

MEZA, Laura; SOZA, Sebastián y VALLE, Paola. Propuesta de un modelo de gestión del riesgo agroclimático. [En línea]. Santiago, Ministerio de Agricultura, FAO. 2011. Disponible en: http://www. fao.org/3/a-as427s.pdf.

MORALES, Bárbara. Acción colectiva y nuevas formas de participación en el marco del conflicto socioambiental. Análisis del proceso experimentado por la Agrupación ciudadana "Por un Pichilemu Limpio", VI Región, Chile. Santiago, Chile. 2009. Tesis Universidad de Chile Facultad de Ciencias Sociales.

PUMARINO Lorena. Catastro y evaluación de necesidades energéticas en comunidades costeras rurales (algueros) de la VI Región. Valparaíso, Universidad Federico Santa María. 2005. Tesis de Licenciatura en Ciencias Ambientales.
RODRÍGUEZ, Adrián y MENESES, Javier. Transformaciones rurales en América Latina y sus relaciones con la población rural. Santiago, CEPAL. 2011.

SEPÚLVEDA, Sergio; RODRÍGUEZ, Adrián; ECHEVERRI, Rafael y PORTILLA, Melania. El enfoque territorial del desarrollo rural. San José, Costa Rica, IICA. 2003.

SERNAPESCA. Anuario estadístico de pesca 2005. Chile, Servicio Nacional de Pesca, Ministerio de Economía, Fomento y Reconstrucción. 2005. ISSN 0716-0976.

SOZA, Sebastián y MEZA, Laura. Gestión del riesgo de sequía y otros eventos climáticos extremos en Chile. Estudio piloto sobre vulnerabilidad y la gestión local del riesgo. Informe Región de O'Higgins. Santiago, FAO. 2010.

SUBDERE. Guía análisis de riesgos naturales para el ordenamiento territorial. SUBDERE. 2011. http:// www.subdere.gov.cl/sites/default/files/documentos/libro_guia_de_analisis_de_riesgos_naturales_para_el_ordenamiento_territorial_.pdf.

UN TEMA de desarrollo: la reducción de la vulnerabilidad frente a los desastres. México, CEPAL, BID. 2000.

WALKER, Brian, HOLLING, C.S., CARPENTER, Stephen R. y KIZING, Ann. Resilience, adaptability and transformability in social-ecological systems. Ecology and Society. 9(2), 2004. ISSN 1708-3087.

WILCHES-CHAUX, Gustavo. Guía de la red para la gestión local del riesgo. Lima, Red de Estudios 